\title{
Specific immune cell and lymphatic vessel signatures identified by image analysis in renal cancer
}

\author{
Peter Schraml ${ }^{1} \cdot$ Maria Athelogou $^{2} \cdot$ Thomas Hermanns $^{3} \cdot$ Ralf Huss $^{2} \cdot$ Holger Moch $^{1}$
}

Received: 21 September 2018 / Revised: 9 January 2019 / Accepted: 19 January 2019 / Published online: 8 February 2019

(c) United States \& Canadian Academy of Pathology 2019

\begin{abstract}
Anti-angiogenic therapy and immune checkpoint inhibition are novel treatment strategies for patients with renal cell carcinoma. Various components and structures of the tumor microenvironment are potential predictive biomarkers and also attractive treatment targets. Macrophages, tumor infiltrating lymphocytes, vascular and lymphatic vessels represent an important part of the tumor immune environment, but their functional phenotypes and relevance for clinical outcome are yet ill defined. We applied Tissue Phenomics methods including image analysis for the standardized quantification of specific components and structures within the tumor microenvironment to profile tissue sections from 56 clear cell renal cell carcinoma patients. A characteristic composition and unique spatial relationship of CD68+ macrophages and tumor infiltrating lymphocytes correlated with overall survival. An inverse relationship was found between vascular (CD34) and lymphatic vessel (LYVE1) density. In addition, outcome was significantly better in patients with high blood vessel density in the tumors, whereas increased lymphatic vessel density in the tumors was associated with worse outcome. The Tissue Phenomics imaging analysis approach allowed visualization and simultaneous quantification of immune environment components, adding novel contextual information, and biological insights with potential applications in treatment response prediction.
\end{abstract}

\section{Introduction}

Metastatic clear cell renal cell carcinoma is a very aggressive disease with poor prognosis. Current treatment strategies include anti-angiogenic therapies with the vascular endothelial growth factor (VEGF)-neutralizing antibody bevacizumab [1] or the tyrosine kinase inhibitors (TKI) sunitinib [2] and sorafenib [3] targeting VEGF and PDGF receptors on tumor cells and intratumoral vessels.

Supplementary information The online version of this article (https:// doi.org/10.1038/s41379-019-0214-z) contains supplementary material, which is available to authorized users.

Holger Moch

holger.moch@usz.ch

1 Department of Pathology and Molecular Pathology, University Hospital Zurich and University Zurich, Schmelzbergstrasse 12, CH-8091 Zurich, Switzerland

2 Definiens AG, Bernhard-Wicki-Str. 5, D-80636 Munich, Germany

3 Department of Urology, University Hospital Zurich and University Zurich, Frauenklinikstrasse 10, CH-8091 Zurich, Switzerland
Pazopanib, another TKI, targets VEGF receptors VEGFR1-VEGFR3 [4]. Immunotherapy recently emerged as additional treatment option in metastatic clear cell renal cell carcinoma [5, 6]. Immune checkpoint inhibitor therapy with antibodies against PD-L1 [7] or PD-1 [8, 9] has been tested in clinical trials. In addition to single agent application, there are already attempts to combine TKI with antiPD-1 [10] or anti-PD-L1 [11] as a combination therapy.

A predictive biomarker is of utmost importance because it helps stratifying renal cell carcinoma (RCC) patients for clinical trials with immunotherapy and anti-angiogenic therapies. In contrast to lung cancer and melanoma patients, predictive biomarkers based on molecular characteristics of the epithelial renal cancer cells are yet to be identified [12]. It has been hypothesized that components of the immune profile of cancer patients can predict therapeutic responses [13, 14]. The effect of the patients' immune system on clinical outcome has important implications for the identification of prognostic markers and biomarkers that predict responses to novel immune therapies or anti-angiogenic therapies.

The tumor microenvironment usually consists of a heterogeneous immune infiltrate potentially including B-cells, 
T-cells, monocytes and macrophages, dendritic cells, mast cells, natural killer (NK) cells, and stromal components such as cancer-associated fibroblasts. Tumor cells grow in a network of vascular and lymphatic vessels $[13,15]$. Tumor infiltrating lymphocytes, tumor-associated macrophages, and blood/lymphatic vessels are not only attractive biomarkers, but also promising therapeutic targets.

In clear cell renal cell carcinoma, the clinical response to VEGF-inhibitors is partly explained by a high density of blood vessels due to the upregulation of VEGF. Tumor tissue of clear cell renal cell carcinoma has a characteristic network of small sinusoidal blood vessels. Previous studies have shown that clear cell renal cell carcinoma with highmicrovessel density (MVD) have better outcome than clear cell renal cell carcinoma with low-MVD [16-18]. This is in contrast to other solid tumors, e.g., breast and bladder cancer $[19,20]$. Recently, experimental data have shown that malignant tumors release growth factors such as VEGF$\mathrm{C}$ to induce lymphatic vessel expansion (lymphangiogenesis) in primary tumors, thereby promoting a lymphatic spread [21]. Although the prognostic relevance of angiogenesis in clear cell renal cell carcinoma has been well documented, the clinical consequence of lymphangiogenesis with intratumoral and peritumoral lymphatic vessels is unclear. This is potentially due to difficulties in identification and quantification of intratumoral lymphatic vessels.

Lymphocytes can be located in the tumor center, in the invasive front of the tumor or in tertiary lymphoid structures around the tumor. Recent analyses of renal cell carcinoma have demonstrated variable numbers of infiltrating immune cells within and around the tumor [22-24]. The immune infiltrates are heterogeneous and very diverse from patient to patient $[13,25,26]$. A high number of tumor infiltrating lymphocytes has been shown to correlate with worse prognosis in clear cell renal cell carcinoma patients [22, 23].

Tumor-associated macrophages are another key immune component in the tumor microenvironment. Distinct subsets of tumor-associated macrophages such as immunostimulatory M1-type macrophages and immunoregulatory M2-type macrophages can either block or facilitate tumor growth, induce or repress antitumor immunity, angiogenesis, and cell migration [27, 28]. However, the presence of macrophages is rarely measured in histological slides. Recently, specific tumor-associated macrophage phenotypes have been identified by mass cytometry and proposed as a potential biomarker in renal cancer [24].

The quantification of tumor infiltrating lymphocytes, tumor-associated macrophages and vessels is difficult in routine histopathology. Intratumoral heterogeneity represents a significant barrier to clinical implementation. Automated image analysis may complement histopathology by standardizing the quantification of potential prognostic or predictive biomarkers of the immune contexture and its spatial relationship [29-31], and can be utilized to extract big data sets from tissue sections to distill additional relevant information.

\section{Materials and methods}

\section{Patients}

A total of 56 patients who underwent surgical treatment of renal cell carcinoma at the University Hospital Zurich were included in this study. Thirty-three of these patients were male and 23 female (median age: 67.5 years). Follow-up data were available from 48 patients (median duration 79 months). The clinico-pathological characteristics of the patient cohort are presented in Supplementary Table 1 . Tumor tissue was evaluated for pathological stage and histological grade according to the 2016 WHO classification and the ISUP grading system [32, 33]. For each tumor, at least one representative tumor tissue block, with a minimum of $1 \mathrm{~cm}$ tumor diameter, was taken for histology. After re-evaluating the hematoxylin and eosin-stained sections, from each case, we selected one block containing the tumor margin with tumor tissue and adjacent nontumorous kidney tissue for image analysis. Histopathologic evaluations of tumor infiltrating lymphocyte densities inside the borders of the tumors were performed by one pathologist (HM) and roughly classified as low, moderate, and high.

This study was approved by the cantonal ethics committee of Zurich (KEK-ZH-No. 2013-0629). The retrospective use of the tumor tissue material is in accordance with the Swiss Law ("Humanforschungsgesetz"), which, according to Article 34, allows the use of biomaterial and patient data for research purposes without informed consent under certain conditions that include the present cases. Law abidance of this study was reviewed and approved by the ethics committee of the Canton Zurich.

\section{Immunohistochemistry}

Formalin fixed and paraffin-embedded sections $(2.5 \mu \mathrm{m})$ were transferred to glass slides followed by immunohistochemical staining according to Ventana (Tucson, AZ, USA) automat protocols. Antibodies and conditions are listed in Supplementary Table 2. Tonsil tissue was used as positive control.

\section{Data analysis}

For data analysis we used Tissue Phenomics, which combines (a) the Definiens Cognition Network Technology (CNT) and the corresponding image data analysis software platforms Definiens Developer XD and Tissue Studio 
(Definiens AG, Munich) with (b) data mining and machine learning methods. In principle, CNT is an artificial intelligence (AI) application, which approximates human ability to interpret objects, patterns, and context in datasets. CNT extends beyond recognizing individual pixels in an image, as it also uses colors, textures, sizes, and spatial relationships to assign pixel groups as individual and hierarchical objects in an image. Structures and features of interest can thus be accurately and robustly segmented and classified within a determined hierarchy created by using the analyzed image [34, 35]. Combining CNT with other AI methods like Data Mining and Machine learning, enables the discovery and extraction of new valuable knowledge and new imagebased biomarkers, which may support scientific insights, diagnosis, progress in patient stratification, prognosis, and patient treatment decision [36, 37].

\section{Image capture and image analysis}

Whole slide images (WSI) were captured on the Zeiss Axio. Scan Z1 tissue slide scanner using a $20 \times$ objective. Dedicated algorithmic solutions were developed using the Definiens Tissue Studio and Developer XD software platform for co-registration and for image analysis. The images analysis results were correlated to the patient clinical outcome data for the identification of potential image-based prognostic biomarkers. Co-registration of patient related WSIs was done by using Definiens Tissue Studio and enables quantification of co-expression for all applied immunohistochemical biomarkers in the same tumor microenvironment. In a next step, immunohistochemically positive single cells were detected, segmented and classified in T-cells (CD3 and CD8), B-cells (CD20), and macrophages (CD68) as well as blood (CD34) and lymphatic vessels (D2-40 and LYVE1). After object detection, segmentation, and classification multiparametric data extraction was achieved using algorithms quantifying absolute, relative, neighborhood's and spatial relationships properties for total numbers, color, and morphological properties of single objects like shape, object groups cell groups and spatial relations between such properties.

\section{Statistical analysis}

Descriptive statistics, correlation, regression, receiver operating characteristic (ROC), and survival analysis were applied after image analysis to quantify the distribution densities and the spatial relationships of T-cells, B-cells, blood-, and lymphatic-vessels related to the tumor microenvironment and to the existing clinical outcome data. Definiens Image Miner New Generation (Definiens AG), the statistical tools (SigmaPlot for windows Version 13.0 (Systat Software, Inc.) and MedCalc Statistical Software version 17.9.7 (MedCalc ${ }^{\circ}$ Software bvba, Ostend, Belgium; http://www.medcalc.org; 2017) were used for further analysis to define and evaluate the prognostic value.

Object distribution densities (population densities) were calculated based on tissue stained areas and on the calculated numbers of single detected, segmented, and classified objects. Using the Definiens Tissue Studio Software the serial WSIs for each individual patient were automatically co-registered, the tumor tissue was manually annotated in a selected WSI and the annotated tissue region was fully automatically transferred into the remaining serial slides. In a next step and for each individual patient the annotated tumor tissue was analyzed using image analysis. Objects like cells and vessels in tumor tissue were detected, segmented, and classified. Numbers and distribution densities of these objects were then quantified in each of the patient's serial tumor tissue slides. These numbers and object distributions in tumor and tumor margins were used as parameters to compare different patient groups. For each parameter, an optimal cut-off value was calculated using ROC analysis. Each cut-off was used to dichotomize the patients into "high" (over the cut-off) and into "low" (below the cut-off) parameter values. This method was applied for each calculated image analysis parameter describing population densities of cells and vessels. The classification results were subsequently used for survival analysis by calculating Kaplan Meier curves and hazard ratios. The cut-off calculation for the distribution densities (vessel areas and object numbers) of lymphatic vessels in LYVE1 stained tumor tissue is shown as an example in Supplementary Figure 2.

The potential impact of each parameter on overall survival (OS) was analyzed using Log-Rank test and CoxRegression models. ImmunoProfiles (IPs) including each investigated parameter were calculated. Radar Plots graphs combining quantitative image analysis results with clinical data were created for the IPs. Furthermore, IPs enable quantitative comparisons between patients and patient groups as well as the assignment of an individual immune status into an appropriate tumor-related "local immune status" group (IPs-group). Similar IPs already known as "cancer immunograms" were described as qualitative visualizations of the state of cancer immune system interactions, which may spur personalized therapy [38]. In the present study, IPs were comparable, "quantitative" descriptions of tumor-related "local immune status".

\section{Results}

\section{Clinical parameters}

We first tested the available pathological parameters for patient stratification. The survival analysis based on the 


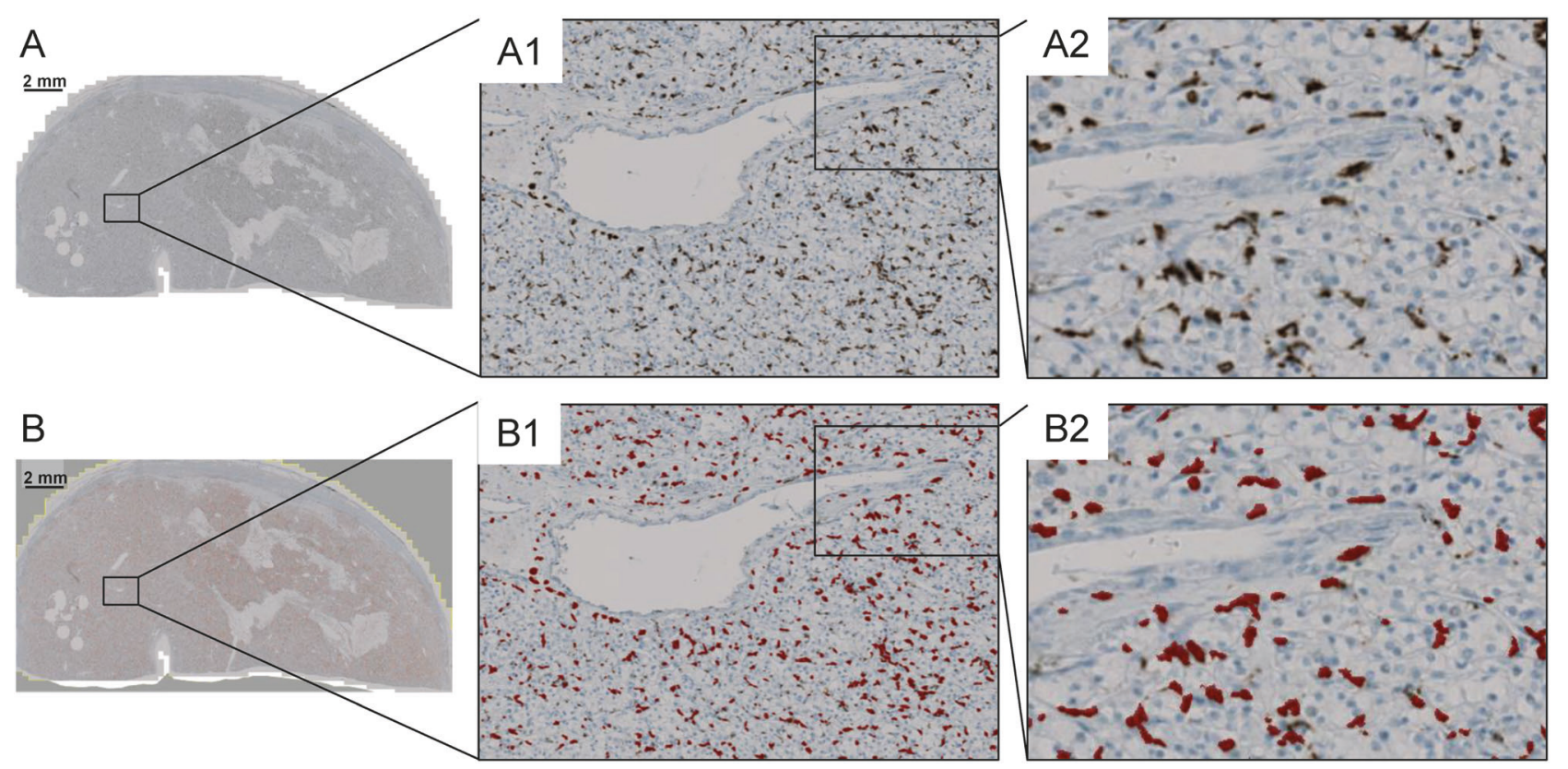

Fig. 1 a Scanned clear cell renal cell carcinoma tissue section immunohistochemically stained against CD68 expressing macrophages with different magnifications of a selected tissue area (A1 and A2). b Image analysis result of the same tissue section with different

ISUP grade [33] and the pathological tumor stage [32] is shown in Supplementary Figure 1. Patients with high-grade tumors (low versus high grade) had a significantly impaired outcome, whereas only a trend was found for late tumor stage.

\section{Characterization of tumor infiltrating lymphocytes, tumor-associated macrophages, blood and lymphatic vessels in clear cell renal cell carcinoma}

Figures 1 and 2 show representative image analysis results from tumor tissue sections with immunostained macrophages (CD68), blood vessels (CD34), and lymphatic vessels (LYVE1). Prior to image analysis, all immunohistochemically stained and scanned sections were validated by one pathologist (HM). Examples for separating tumor from nontumorous areas are shown in Supplementary Figure 3. Using the Definiens Tissue Studio and the Developer XD software platform for image analysis, tumor areas, single-tumor infiltrating lymphocyte objects, single objects of tumor-associated macrophages and single vessel objects were detected, segmented and classified in a fully automated way. Tumor from nontumor tissue areas were separated as defined and the areas were calculated in pixels. The number of the tumor infiltrating lymphocytes, tumorassociated macrophages, blood and lymphatic vessels in the corresponding tumor areas were counted. The image analysis results were automatically exported in CSV tables for further statistical analysis. The purpose of this analysis magnifications of the same selected tissue area (B1 and B2) after detection, segmentation, and classification of CD68-positive macrophages (brown)

was to calculate the stratification power of the image analysis results related to the clinical parameter "overall survival" of the patients. For these purposes the densities of tumor infiltrating lymphocytes, tumor-associated macrophages and vessels in the tumor areas were calculated. These densities were calculated as the number of counted objects divided by the corresponding tumor area. The corresponding tumor areas were calculated in pixels.

As shown in Table 1, cut-offs of population densities were calculated using ROC-analysis. The Supplementary Figure 2 shows representative examples for the cut-off calculations based on the ROC analysis. The calculated cutoffs allowed the separation of the entire patient group into two subgroups (see Table 1). One subgroup consisted of those patients shown high and the other subgroup consisted of those patients shown low-population densities of tumor infiltrating lymphocytes, tumor-associated macrophages, blood and lymphatic vessels. The calculated median values of object densities for each of these subgroups and the number of analyzed patient cases are shown in Table 1, too.

In a next step the calculated cut-offs and the corresponding object densities, were used to quantify the "power" of image analysis results for patient stratification. For this purpose the object population densities were used as parameters for the stratification of the patients related to the clinical parameter "Overall Survival". Kaplan Meier curves, the corresponding $p$ values and hazard ratios were calculated for tumor infiltrating lymphocytes, tumor-associated macrophages, blood and lymphatic vessel population densities. 

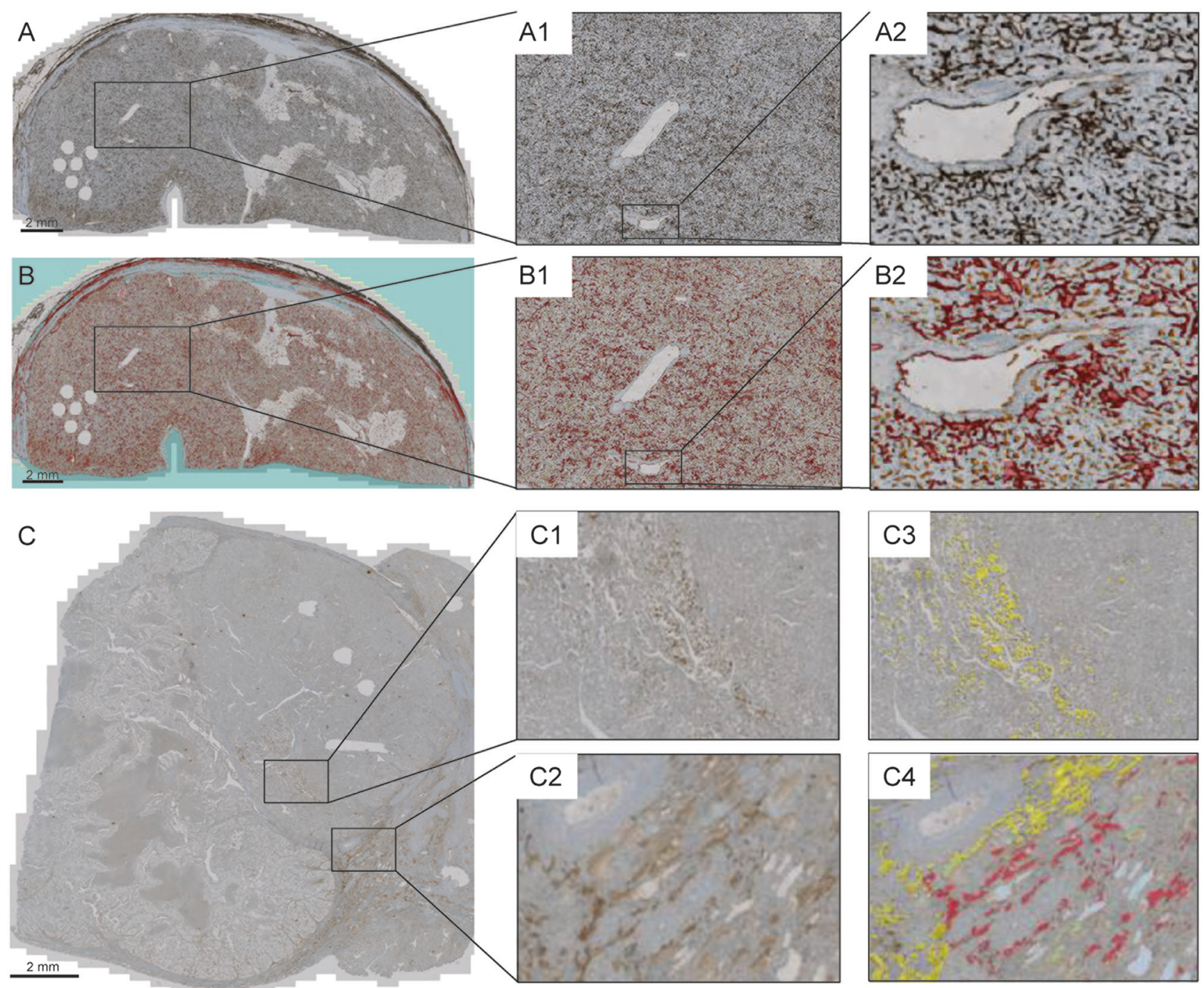

Fig. 2 Consecutive tissue section of the same clear cell renal cell carcinoma tissue shown in Fig. 1 immunohistochemically stained against CD34 expressing blood vessels (a). Detected, segmented, and classified blood vessels (b). Tissue section of another clear cell renal cell carcinoma with immunohistochemically stained lymphatic vessels (LYVE1) (c). Magnified tissue areas with stained (A1 and A2) and marked blood vessels after detection, segmentation, and classification. Magnified areas of stained (C1 and C2) and marked lymphatic vessels (C3 and C4). Intratumoral lymphatic vessels are marked in yellow, lymphatic vessels detected in the tumor microenvironment are marked in red
As shown in in Table 2 and Fig. 3, statistical significance and high hazard ratios for poor clinical outcome were associated with low-T cell (CD3 and CD8), high-B cell (CD20), and high-macrophage (CD68) population densities. Patients with high-blood vessel (CD34) density in the tumors had a significantly better outcome and, conversely, patients with high-lymphatic vessel density in the tumors had a significantly impaired outcome. This finding held true for both intratumoral lymphatic vessels and intra-/extratumoral lymphatic vessels. As expected, almost all D2-40 and LYVE1-positive vessels were negative for CD34 and most CD34-positive vessels were negative for D2-40 and LYVE1.

\section{Assigning IPs to pathological parameters using radar plots}

As shown in Fig. 4, radar plots were calculated using the median values of the calculated object densities. These plots visualize the "quantified patient local tumor-related immune profile-IP" related to prominent clinical parameters like those for OS (alive and dead) and for tumor grade. All density values were normalized to the maximal value 1 . As shown in Fig. 4a there was a positive correlation between the presence of high-blood vessel density and better outcome. It is obvious that the "simple visual inspection" of the radar plots show that, patients with longer survival and low 
Table 1 Median values of population densities in clear cell renal cell carcinoma and number of tumors with low and high densities of tumor infiltrating lymphocytes, tumor-associated macrophages, blood and lymphatic vessels

\begin{tabular}{llllllll}
\hline & CD3 & CD8 & CD20 & CD68 & CD34 & D2-40 & LYVE1 \\
\hline Cut-off population density & 0.005006552 & 0.004607 & 0.0043022 & 0.006259 & 0.08973 & 0.000043553 & 0.000907856 \\
Median population density & 0.005063 & 0.004946 & 0.003714 & 0.006259 & 0.1438 & 0.0001129 & 0.001135 \\
Median tumors with low density & 0.00422 & 0.00404 & 0.00357 & 0.00539 & 0.0640 & 0.0000259 & 0.000445 \\
Median tumors with high density & 0.00565 & 0.00562 & 0.00432 & 0.00772 & 0.154 & 0.000152 & 0.00435 \\
Number of analyzable cases & 51 & 47 & 48 & 51 & 49 & 45 & 37 \\
\hline
\end{tabular}

Population densities of T-cells (CD3 and CD8), B-cells (CD20), macrophages (CD68), lymphatic vessels (D2-40 and LYVE1) were calculated using object numbers. Population densities of blood vessels (CD34) were determined by area

grade tumors showed different IPs as those patients with short survival and high-grade tumors (Fig. 4b). According to these results, we suggest that the use of radar plots represent a potential supportive diagnostic tool for cancer immunotherapy.

\section{Discussion}

In this study, an automated image analysis system with standardization and fully reproducible quantification of immune contexture features was able to identify immune cell and vessel characteristics as part of a complex ecosystem in renal cancer. Presence of tiny intratumoral lymphatic vessels and increased macrophage number in the primary tumor were associated with poor patient outcome.

We demonstrated that high densities of T-cells in renal cancer are associated with better outcome, whereas a high density of B-cells has a negative impact on survival. The effect that the density of T-cells correlates with better prognosis in renal cancer is an important result that deserves further analysis.

Histologically, tumors can be categorized as inflamed or noninflamed [26, 39]. Inflamed ("hot") tumors are characterized by the presence of tumor infiltrating lymphocytes with a high density of CD8-positive T-cells and the presence of a pre-existing antitumor immune response. Noninflamed ("cold") tumors are immunologically "ignorant" and are usually poorly infiltrated by lymphocytes ("deserted") or lymphocytes still remain outside the tumor tissue ("excluded") and therefore prevented or incapable to engage with cancer cells.

The fact that functional populations of immune cells have different effects in different cancer types suggests that the role of the immune cell population in tumor control varies between cancer types. Correlations between the levels of immune cell infiltration and outcome in RCC are controversial. The density of CD8-positive T-cells correlated with poor prognosis in RCC, except when these cells are proliferating [22]. A strong lymphocytic infiltration has also been reported to be associated with better clinical outcome of clear cell renal cell carcinoma patients, as well as in patients with bladder cancer, breast cancer, and other tumors [40]. Future imaging studies should therefore not only quantify tumor infiltrating lymphocytes, but also characterize different functional populations of immune cells in clear cell renal cell carcinoma [24, 41-44].

More importantly, we demonstrate in tissue sections that increased density of tumor-associated macrophages is associated with poor clinical outcome. Tumor-associated macrophage phenotypes are heterogeneous among tumors and recent reports distinguish between antitumor M1 and protumor M2 subtypes. The presence of M2 macrophages favors tumor growth and spreading [45-47]. A previous study identified 17 tumor-associated macrophage phenotypes as well as 22 different T-cell phenotypes [24] in clear cell renal cell carcinoma. These distinct immune compositions correlated with survival of renal cancer patients. To further understand the functional diversity of tumorassociated macrophage phenotypes, the relationship with other immune cell populations needs to be investigated.

Our analysis revealed that induction of intratumoral lymphangiogenesis is a major biomarker for poor prognosis in clear cell renal cell carcinoma. Whereas microvascular density has been frequently examined in RCC, formation of intratumoral lymphangiogenesis has received little attention in the past. Vascular vessels are mainly analyzed and quantified by immunostaining using anti-CD34 antibodies [18]. However, it is important to note that RCC vascularity assessed by CD34 staining also includes lymphatic vessels. Recent studies have shown that about 50\% of tumorassociated lymphatic vessels are also CD34-positive [48]. Antibodies that specifically recognize human podoplanin (D2-40) and the lymphatic vessel endothelia receptor 1 (LYVE1) can be used to differentiate lymphatic vessels from vascular vessels in paraffin-embedded sections [49-54]. The combination of image analysis and immunohistochemical allowed us to identify tiny D2-40- and LYVE1-positive lymphatic vessels in the intratumoral and peritumoral areas of RCC. The clinical significance of peritumoral lymphatic vessels and intratumoral lymphatic vessels as parameters of lymphangiogenesis is 
Table 2 Results after receiver operating characteristic (ROC) and survival analysis for the population densities of T-, Bcells, tumor-associated macrophages, blood and lymphatic vessels

\begin{tabular}{llllll}
\hline & Cut-off & Poor outcome & Hazard ratio (95\% CI) & 95\% CI & $p$ Value \\
\hline CD3 density & 0.005006552 & Low & 3.4957 & $1.2210-10.0079$ & 0.0144 \\
CD8 density & 0.00460718 & Low & 2.6867 & $0.8271-8.7279$ & 0.0528 \\
CD20 density & 0.004302238 & High & 2.7374 & $0.6768-11.0722$ & 0.0630 \\
CD68 density & 0.006259409 & High & 3.6307 & $1.3067-10.0881$ & 0.0172 \\
CD34 density & 0.089737708 & Low & 3.7110 & $0.6289-21.8975$ & 0.0153 \\
D2-40 density & 0.000043553 & High & 5.0302 & $1.5644-16.1742$ & 0.0819 \\
LYVE1 density & 0.000907856 & High & 4.2281 & $1.6206-11.0313$ & 0.0057 \\
\hline
\end{tabular}

Density calculations were done based on the number of single T-, B-cells, tumor-associated macrophages, and lymphatic vessels. The density for blood vessels was determined by the covered area controversial. In different tumors, poor survival in patients with presence of intratumoral lymphatic vessels has been reported $[49,50,55,56]$. Our results are consistent with such findings in other tumors and suggest that intratumoral lymphangiogenesis is probably a major route by which renal cancer metastasize [21]. We used tissue from the tumor/nontumor tissue border for our analysis, because the highest number of tumor infiltrating lymphocytes is usually observed at the invasive margin of a tumor. Combined measurement of intratumoral and peritumoral lymphatic vessels increased the prognostic impact of lymphatic vessels, suggesting that interaction of tumor infiltrating lymphocytes and lymphatic vessels in this tumor compartment is relevant for tumor progression.

The stimulus for lymphangiogenesis can be triggered by tumor-related and/or inflammatory-related processes [21]. Clear cell renal cell carcinoma as well as macrophages can produce growth factors, such as vascular endothelial growth factor C (VEGF-C) [57, 58], known to induce lymphangiogenesis. In his seminal study, Bonsib [57] has demonstrated the presence of small clusters of newly formed lymphatics within inflamed renal sinus outside a pseudocapsule and at the invasive front, but not within the tumor. Although Bonsib has observed intralymphatic tumor cells in peritumoral lymphatics of clear cell renal cell carcinoma, lymphatic spread to locoregional lymph nodes is rare in this tumor subtype [59]. It is more common in papillary and translocation renal cell carcinoma [60,61].

Whereas intratumoral MVD of the primary clear cell renal cell carcinoma is associated with good prognosis, it has been shown that extrarenal renal sinus vein involvement is the most important route for hematogeneous metastasis in clear cell renal cell carcinoma [62]. Therefore, renal sinus vein involvement conveys adverse prognostic importance. In our study, we observed newly formed intratumoral and peritumoral lymphatic vessels, which indicate poor patient prognosis. This finding is difficult to explain in light of the knowledge that clear cell renal cell carcinoma primarily shows hematogeneous metastasis [47]. Bonsib has hypothesized that the impact of intralymphatic tumor cells to predict poor prognosis could relate to hematogeneous spread rather than lymphatic spread if lymphatic vessels sprouted from the venous system in the peritumoral area, which is much more frequently involved by tumor [57]. The concept of newly formed lymphatics sprouting from venous vessels is debated, but there are other current concepts which could explain the prognostic impact of newly formed lymphatic vessels in clear cell renal cell carcinoma: (i) it has been shown that tumor-associated lymphatic vessels specifically inhibit tumor-exiting, antigen-experienced T-cells. Dieterich et al. [63] demonstrated that primary tumorassociated lymphatic vessels express the T-cell inhibitory protein PD-L1, which may be involved in the inhibition of tumor-emigrating T-cells. (ii) Stem-like tumor cells have also been found to reside in the vicinity of lymphatic vessels, suggesting that there is a "lymphovascular" stem cell niche [58]. (iii) Recently, Qiaoli et al. have elegantly shown in melanoma that tumor lymphangiogenesis occurs also in established metastases of distant organs. This unexpected contribution of lymphatics in distant organs could promote further metastatic spread of those metastases to other organs [64].

Tumor infiltrating lymphocytes, tumor-associated macrophages, and intratumoral lymphatics have potential prognostic significance in different tumors [49, 50, 55, 65] and represent potential treatment targets [66]. The semiquantification of tumor infiltrating lymphocytes and intratumoral vessels in renal cancer suffers from poor reproducibility and inter- and intra-observer variability. In keeping with this, a series of our semiquantified tumors with low- and high-inflammatory infiltrates correlated with low and high $\mathrm{CD} 3, \mathrm{CD} 8$, and $\mathrm{CD} 20$ cell population density by image analysis. However, rather discrepant results were particularly obtained from tumors with moderate lymphocyte density (data not shown) suggesting the microscopic evaluation of intermediate lymphocyte infiltrates is rather subjective as the total number of single lymphocytes present in tumors may often be underestimated. Image analysis offers the standardization and the fully reproducible quantification of such features. Especially the small intratumoral 

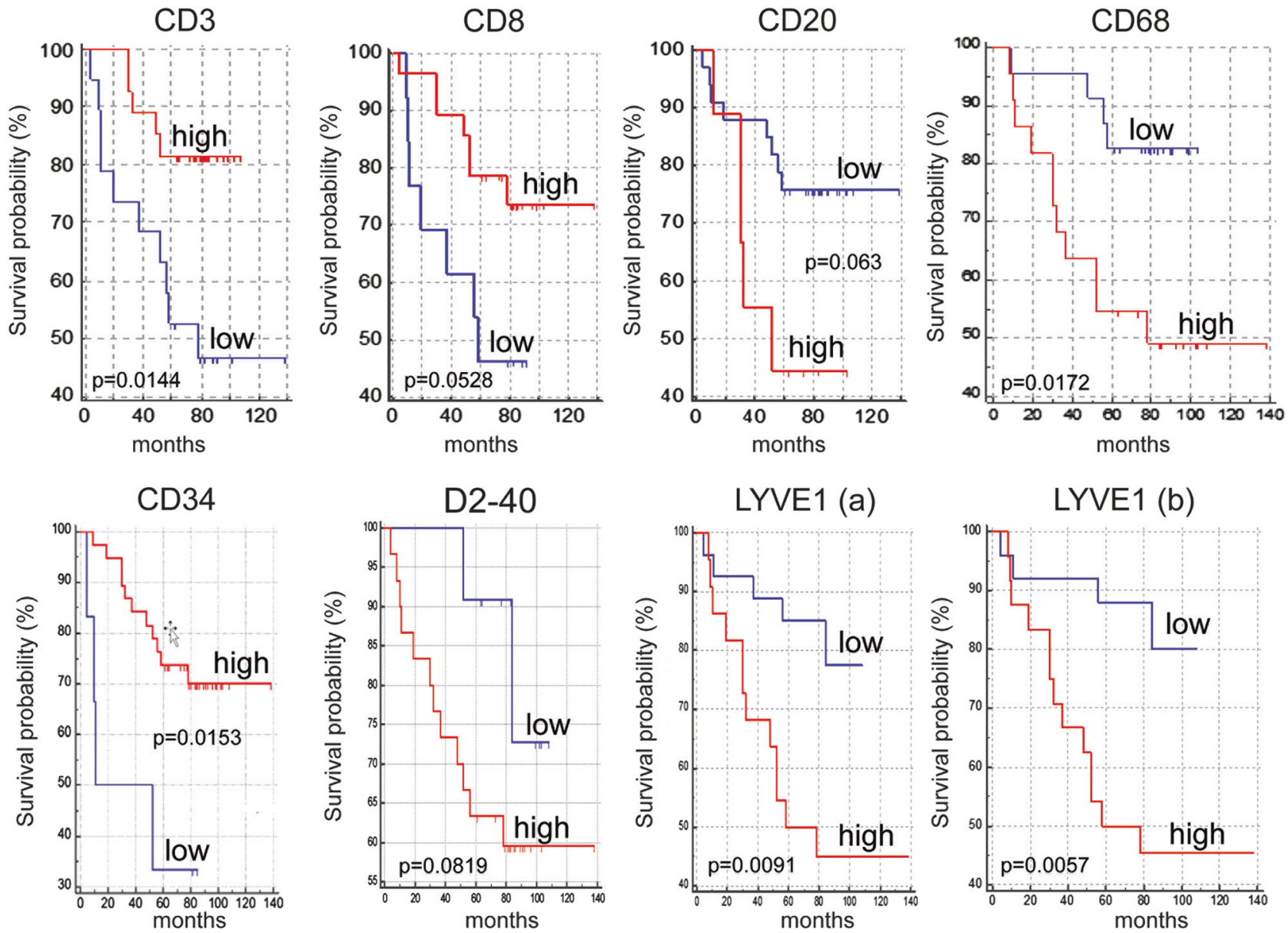

Fig. 3 Kaplan Meier plots for CD3, CD8, CD20, CD68, CD34, D2-40, and LYVE1 population densities based on the number of single cells or area (CD34). Poor clinical outcome is related to low-T cell, high-B cell, high tumor-associated macrophages, low-blood

lymphatic vessels may proof particularly obscure under classical HE stained tissue. Intratumoral vessels and their relationship to tumor infiltrating lymphocytes and tumorassociated macrophages can be quantified with our image analysis algorithm making it amenable to most clinical laboratories. This translational impact would rely on the wide adoption of digital pathology in clinical routine, which is possible in the near future. Targeted treatments such as TKI and novel checkpoint immune therapies should be guided by standardized and robust biomarkers. We demonstrate the value of an imaging approach using clear cell renal cell carcinoma as an example. Our study further suggests a link between a tumor-associated macrophage phenotype, a specific composition of tumor infiltrating lymphocytes and an angiogenic switch from a high-MVD of vascular vessels to expansion of lymphatic vessel in primary tumors that is associated with poor outcome.

In the future, the development of imaging analysis tools in the context of Tissue Phenomics for standardized measurement of lymphocyte infiltration, macrophage vessel, and high-lymphatic vessel population densities. LYVE1 a intratumoral lymphatic vessels; LYVE1 b intra- and extratumoral lymphatic vessels combined

quantification and vessel analysis may allow a more precise measurement of the immune contexture in renal cell cancer. These factors need also to be taken into consideration while interpreting contextual data from tissues derived from metastases after treatment with anti-angiogenic therapy or checkpoint inhibitors. The comprehensive analysis of the interactions between three complex systems-the tumor cells, the immune response and the tissue microenvironment with blood and lymphatic vessels requires the use of systems biology approaches to integrate tumor cell associated, immune cell associated and microenvironment-associated parameters with clinical data.

In summary, virtual multiplexing combined with multimodal stain will enable the quantification of spatial relations of all positively stained objects from consecutive WSI and will provide additional value of the immune contexture quantification for renal cancer. This approach will allow developing a scheme of the dynamic actions of tumor cells with the immune system in the future and thus can help to stratify patients for effective treatment options. 


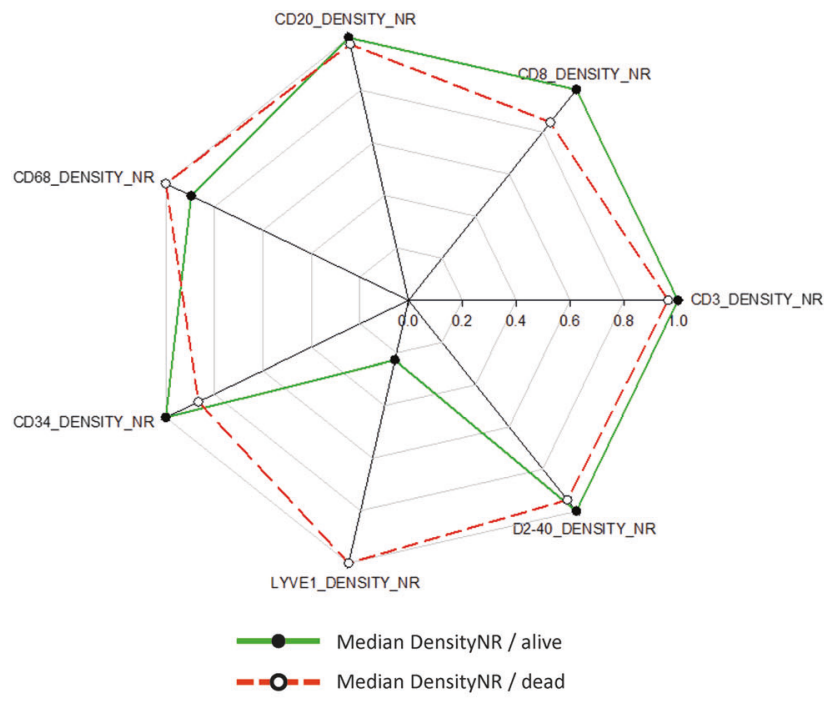

Fig. 4 Radar Plot visualization for the median values of image analysis results quantifying the tumor infiltrating lymphocytes, tumor-

Acknowledgments We thank Susanne Dettwiler and Fabiola Prutek from the tissue biobank and Christiane Mittmann from the in situ laboratory of the Department of Pathology and Molecular Pathology, University Hospital Zurich, for generating and immunostaining RCC tissue sections. This work was supported by the Swiss National Science Foundation under Grant number S-87701-03-01 (HM).

\section{Compliance with ethical standards}

Conflict of interest The authors declare that they have no conflict of interest. HM is scientific advisory board member of Definiens AG.

Publisher's note: Springer Nature remains neutral with regard to jurisdictional claims in published maps and institutional affiliations.

\section{References}

1. Escudier B, Pluzanska A, Koralewski P, et al. Bevacizumab plus interferon alfa-2a for treatment of metastatic renal cell carcinoma: a randomised, double-blind phase III trial. Lancet. 2007;370:2103-11.

2. Motzer RJ, Hutson TE, Tomczak P, et al. Sunitinib versus interferon alfa in metastatic renal-cell carcinoma. N Engl J Med. 2007;356:115-24.

3. Escudier B, Eisen T, Stadler WM, et al. Sorafenib in advanced clear-cell renal-cell carcinoma. N Engl J Med. 2007;356:125-34.

4. Sternberg CN, Davis ID, Mardiak J, et al. Pazopanib in locally advanced or metastatic renal cell carcinoma: results of a randomized phase III trial. J Clin Oncol. 2010;28:1061-8.

5. Kucharczyk J, Matrana MR, Santoni M, et al. Emerging immunotargets in metastatic renal cell carcinoma. Curr Drug Targets. 2016;17:771-6.

6. Mann SA, Lopez-Beltran A, Massari F, et al. Targeting the programmed cell death-1 pathway in genitourinary tumors: current progress and future perspectives. Curr Drug Metab. 2017;18:700-11.

7. McDermott DF, Sosman JA, Sznol M, et al. Atezolizumab, an anti-programmed death-ligand 1 antibody, in metastatic renal cell

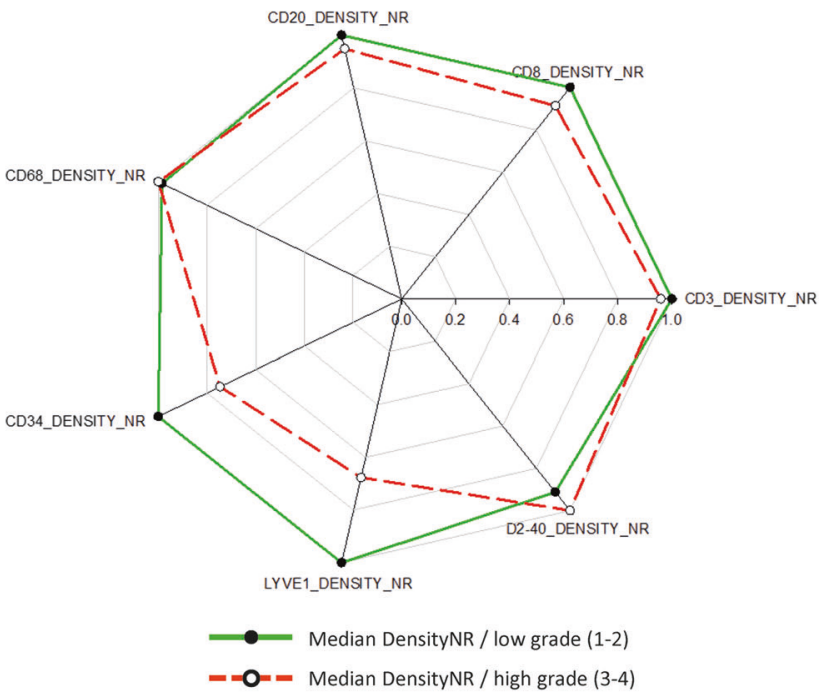

associated macrophages, blood and lymphatic vessel populations grouped according to survival (left) and ISUP grade (right)

carcinoma: long-term safety, clinical activity, and immune correlates from a phase IA study. J Clin Oncol. 2016;34:833-42.

8. Motzer RJ, Escudier B, McDermott DF, et al. Nivolumab versus everolimus in advanced renal-cell carcinoma. $\mathrm{N}$ Engl $\mathrm{J}$ Med. 2015;373:1803-13.

9. Topalian SL, Hodi FS, Brahmer JR, et al. Safety, activity, and immune correlates of anti-PD-1 antibody in cancer. N Engl J Med. 2012;366:2443-54.

10. Amin A, Plimack ER, Infante JR, et al. Nivolumab (anti-PD-1; BMS-936558, ONO-4538) in combination with sunitinib or pazopanib in patients with metastatic renal cell carcinoma (mRCC). BJU Int. 2014;114:2.

11. Choueiri TK, Plimack ER, Gupta $S$, et al. Phase lb dose-finding study of axitinib plus pembrolizumab in treatment-naive patients with advanced renal cell carcinoma. BJU Int. 2015;116:5.

12. Tan PH, Cheng L, Rioux-Leclercq N, et al. Renal tumors: diagnostic and prognostic biomarkers. Am J Surg Pathol. 2013;37:1518-31.

13. Fridman WH, Pages F, Sautes-Fridman C, et al. The immune contexture in human tumours: impact on clinical outcome. Nat Rev Cancer. 2012;12:298-306.

14. Galon J, Fridman WH, Pages F. The adaptive immunologic microenvironment in colorectal cancer: a novel perspective. Cancer Res. 2007;67:1883-6.

15. Schreiber RD, Old LJ, Smyth MJ. Cancer immunoediting: integrating immunity's roles in cancer suppression and promotion. Science. 2011;331:1565-70.

16. Schraml P, Struckmann K, Hatz F, et al. VHL mutations and their correlation with tumour cell proliferation, microvessel density, and patient prognosis in clear cell renal cell carcinoma. J Pathol. 2002;196:186-93.

17. Jilaveanu LB, Puligandla M, Weiss SA, et al. Tumor microvessel density as a prognostic marker in high-risk renal cell carcinoma patients treated on ECOG-ACRIN E2805. Clin Cancer Res. 2018;24:217-23.

18. Mertz KD, Demichelis F, Kim R, et al. Automated immunofluorescence analysis defines microvessel area as a prognostic parameter in clear cell renal cell cancer. Hum Pathol. 2007;38:1454-62. 
19. Bochner BH, Cote RJ, Weidner N, et al. Angiogenesis in bladdercancer-relationship between microvessel density and tumor prognosis. J Natl Cancer Inst. 1995;87:1603-12.

20. Weidner N, Gasparini G, Bevilacqua P, et al. Tumor microvessel density, P53 expression and tumor size are relevant prognostic markers in node-negative breast-carcinoma. Lab Invest. 1994;70: A24.

21. Karaman S, Detmar M. Mechanisms of lymphatic metastasis. J Clin Invest. 2014;124:922-8.

22. Nakano O, Sato M, Naito Y, et al. Proliferative activity of intratumoral CD8(+) T-lymphocytes as a prognostic factor in human renal cell carcinoma: clinicopathologic demonstration of antitumor immunity. Cancer Res. 2001;61:5132-6.

23. Jensen HK, Donskov F, Nordsmark M, et al. Increased intratumoral FOXP3-positive regulatory immune cells during interleukin-2 treatment in metastatic renal cell carcinoma. Clin Cancer Res. 2009;15:1052-8.

24. Chevrier S, Levine JH, Zanotelli VRT, et al. An immune Atlas of clear. Cell Ren Cell Carcinoma Cell. 2017;169:736-49.

25. Galon J, Mlecnik B, Bindea G, et al. Towards the introduction of the 'Immunoscore' in the classification of malignant tumours. $\mathrm{J}$ Pathol. 2014;232:199-209.

26. Hegde PS, Karanikas V, Evers S. The where, the when, and the how of immune monitoring for cancer immunotherapies in the era of checkpoint inhibition. Clin Cancer Res. 2016;22:1865-74.

27. Laoui D, Movahedi K, Van Overmeire E, et al. Tumor-associated macrophages in breast cancer: distinct subsets, distinct functions. Int J Dev Biol. 2011;55:861-7.

28. Sakakura K, Takahashi H, Kaira K, et al. Relationship between tumor-associated macrophage subsets and CD47 expression in squamous cell carcinoma of the head and neck in the tumor microenvironment. Lab Invest. 2016;96:994-1003.

29. Caie PD, Turnbull AK, Farrington SM, et al. Quantification of tumour budding, lymphatic vessel density and invasion through image analysis in colorectal cancer. J Transl Med. 2014;12:156.

30. Caie PD, Zhou Y, Turnbull AK, et al. Novel histopathologic feature identified through image analysis augments stage II colorectal cancer clinical reporting. Oncotarget. 2016; 7:44381-94.

31. Heindl A, Nawaz S, Yuan Y. Mapping spatial heterogeneity in the tumor microenvironment: a new era for digital pathology. Lab Invest. 2015;95:377-84.

32. Moch H, Humphrey PA, Ulbright TM, et al. WHO Classification of Tumours of the Urinary System and Male Genital Organs. Lyon, France: International Agency for Research on Cancer; 2016.

33. Delahunt B, Cheville JC, Martignoni G, et al. The International Society of Urological Pathology (ISUP) grading system for renal cell carcinoma and other prognostic parameters. Am J Surg Pathol. 2013;37:1490-504.

34. Athelogou M, Schmidt, G, Schäpe, A, Baatz, M, Binnig, G. Cognition Network Technology-A Novel Multimodal Image Analysis Technique for Automatic Identification and Quantification of Biological Image Contents. Berlin, Heidelberg: SpringerVerlag; 2007.

35. Binnig G. Context Driven Image Analys is in Tissue Phenomics, Profiling Cancer Patients for Treatment Decisions. Vol. 1. Berlin, Heidelberg: Springer-Verlag; 2018. p. 35-65.

36. Athelgou M, Huss, R. Tissue Phenomics, Profiling Cancer Patients for Treatment Decisions. Vol 1. Berlin Heidelberg, 2018. p. $175-82$.

37. Harder N, Athelogou M, Hessel H, et al. Tissue phenomics for prognostic biomarker discovery in low- and intermediate-risk prostate cancer. Sci Rep. 2018;8:4470.
38. Blank CU, Haanen JB, Ribas A, et al. CANCER IMMUNOLOGY. The "cancer immunogram". Science. 2016;352:658-60.

39. Sharma P, Allison JP. The future of immune checkpoint therapy. Science. 2015;348:56-61.

40. Gooden MJ, de Bock GH, Leffers N, et al. The prognostic influence of tumour-infiltrating lymphocytes in cancer: a systematic review with meta-analysis. $\mathrm{Br} \mathrm{J}$ Cancer. 2011;105:93-103.

41. Baine MK, Turcu G, Zito CR, et al. Characterization of tumor infiltrating lymphocytes in paired primary and metastatic renal cell carcinoma specimens. Oncotarget. 2015;6:24990-45002.

42. Barnes TA, Amir E. HYPE or HOPE: the prognostic value of infiltrating immune cells in cancer. $\mathrm{Br} \mathrm{J}$ Cancer. 2017; 117:451-60.

43. Geissler K, Fornara P, Lautenschlager C, et al. Immune signature of tumor infiltrating immune cells in renal cancer. Oncoimmunology 2015;4:e985082-1.

44. Giraldo NA, Becht E, Vano Y, et al. Tumor-infiltrating and peripheral blood $\mathrm{T}$-cell immunophenotypes predict early relapse in localized clear cell renal cell carcinoma. Clin Cancer Res. 2017;23:4416-28

45. Sconocchia G, Spagnoli GC, Del Principe D, et al. Defective infiltration of natural killer cells in MICA/B-positive renal cell carcinoma involves beta(2)-integrin-mediated interaction. Neoplasia. 2009;11:662-71.

46. Dannenmann SR, Thielicke J, Stockli M, et al. Tumor-associated macrophages subvert T-cell function and correlate with reduced survival in clear cell renal cell carcinoma. Oncoimmunology. 2013;2:e23562.

47. Wyler L, Napoli CU, Ingold B, et al. Brain metastasis in renal cancer patients: metastatic pattern, tumour-associated macrophages and chemokine/chemoreceptor expression. Br J Cancer. 2014;110:686-94.

48. Fiedler U, Christian S, Koidl S, et al. The sialomucin CD34 is a marker of lymphatic endothelial cells in human tumors. Am J Pathol. 2006;168:1045-53.

49. Cueni LN, Hegyi I, Shin JW, et al. Tumor lymphangiogenesis and metastasis to lymph nodes induced by cancer cell expression of podoplanin. Am J Pathol. 2010;177:1004-16.

50. Dadras SS, Lange-Asschenfeldt B, Velasco P, et al. Tumor lymphangiogenesis predicts melanoma metastasis to sentinel lymph nodes. Mod Pathol. 2005;18:1232-42.

51. Schacht V, Dadras SS, Johnson LA, et al. Up-regulation of the lymphatic marker podoplanin, a mucin-type transmembrane glycoprotein, in human squamous cell carcinomas and germ cell tumors. Am J Pathol. 2005;166:913-21.

52. Bono P, Wasenius VM, Heikkila P, et al. High LYVE-1-positive lymphatic vessel numbers are associated with poor outcome in breast cancer. Clin Cancer Res. 2004;10:7144-9.

53. Gao F, Lu YM, Cao ML, et al. Expression and quantification of LYVE-1 in human colorectal cancer. Clin Exp Med. 2006;6:65-71.

54. Kato T, Prevo R, Steers G, et al. A quantitative analysis of lymphatic vessels in human breast cancer, based on LYVE-1 immunoreactivity. Br J Cancer. 2005;93:1168-74.

55. Stacker SA, Achen MG, Jussila L, et al. Lymphangiogenesis and cancer metastasis. Nat Rev Cancer. 2002;2:573-83.

56. Tobler NE, Detmar M. Tumor and lymph node lymphangiogenesis-impact on cancer metastasis. J Leukoc Biol. 2006;80:691-6.

57. Bonsib SM. Renal lymphatics, and lymphatic involvement in sinus vein invasive (pT3b) clear cell renal cell carcinoma: a study of 40 cases. Mod Pathol. 2006;19:746-53.

58. Ma Q, Dieterich LC, Detmar M. Multiple roles of lymphatic vessels in tumor progression. Curr Opin Immunol. 2018; $53: 7-12$. 
59. Baldewijns MM, Roskams T, Ballet V, et al. A low frequency of lymph node metastasis in clear-cell renal cell carcinoma is related to low lymphangiogenic activity. BJU Int. 2009;103:1626-31.

60. Renshaw AA, Richie JP. Subtypes of renal cell carcinoma. Different onset and sites of metastatic diseas. Differ onset sites metastatic Dis Am J Clin Pathol. 1999;111:539-43.

61. Argani P, Olgac S, Tickoo SK, et al. Xp11 translocation renal cell carcinoma in adults: expanded clinical, pathologic, and genetic spectrum. Am J Surg Pathol. 2007;31:1149-60.

62. Bonsib SM. The renal sinus is the principal invasive pathway: a prospective study of 100 renal cell carcinomas. Am J Surg Pathol. 2004;28:1594-1600.
63. Dieterich LC, Ikenberg K, Cetintas T, et al. Tumor-associated lymphatic vessels upregulate PDL1 to inhibit T-cell activation. Front Immunol. 2017;8:66.

64. Ma Q, Dieterich LC, Ikenberg K, et al. Unexpected contribution of lymphatic vessels to promotion of distant metastatic tumor spread. Sci Adv. 2018;4:eaat4758.

65. Horiguchi A, Ito K, Sumitomo M, et al. Intratumoral lymphatics and lymphatic invasion are associated with tumor aggressiveness and poor prognosis in renal cell carcinoma. Urology. 2008;71:928-32.

66. Wissmann C, Detmar M. Pathways targeting tumor lymphangiogenesis. Clin Cancer Res. 2006;12:6865-8. 